\title{
Serotype-specific differences in short- and longer-term mortality following invasive pneumococcal disease
}

\author{
G. J. HUGHES ${ }^{1}$, L. B. WRIGHT ${ }^{2}$, K. E. CHAPMAN ${ }^{2}$, D. WILSON ${ }^{2}$ AND \\ R. GORTON ${ }^{2} *$ \\ ${ }_{2}^{1}$ Academic Unit of Public Health, University of Leeds, Leeds, UK \\ ${ }^{2}$ Public Health England North East, Citygate, Gallowgate, Newcastle upon Tyne, UK
}

Received 17 November 2015; Final revision 16 March 2016; Accepted 13 April 2016; first published online 19 May 2016

\section{SUMMARY}

Invasive pneumococcal disease (IPD), caused by infection with Streptococcus pneumoniae, has a substantial global burden. There are over 90 known serotypes of $S$. pneumoniae with a considerable body of evidence supporting serotype-specific mortality rates immediately following IPD. This is the first study to consider the association between serotype and longer-term mortality following IPD. Using enhanced surveillance data from the North East of England we assessed both the short-term (30-day) and longer-term ( $\leqslant 7$ years) independent adjusted associations between individual serotypes and mortality following IPD diagnosis using logistic regression and extended Cox proportional hazards models. Of the 1316 cases included in the analysis, 243 [18.5\%, 95\% confidence interval (CI) 16.4-20.7] died within 30 days of diagnosis. Four serotypes $(3,6 \mathrm{~A}, 9 \mathrm{~N}, 19 \mathrm{~F})$ were significantly associated with overall increased 30-day mortality. Effects were observable only for older adults ( $\geqslant 60$ years). After extension of the window to 12 months and 36 months, one serotype was associated with significantly increased mortality at 12 months (19 F), but no individual serotypes were associated with increased mortality at 36 months. Two serotypes had statistically significant hazard ratios (HR) for longer-term mortality: serotype 1 for reduced mortality (HR $0 \cdot 51,95 \%$ CI $0 \cdot 30-0 \cdot 86$ ) and serotype $9 \mathrm{~N}$ for increased mortality (HR $2 \cdot 30,95 \%$ CI $1 \cdot 29-4 \cdot 37$ ). The association with serotype $9 \mathrm{~N}$ was no longer observed after limiting survival analysis to an observation period starting 30 days after diagnosis. This study supports the evidence for associations between serotype and short-term (30-day) mortality following IPD and provides the first evidence for the existence of statistically significant associations between individual serotypes and longer-term variation in mortality following IPD.

Key words: Mortality, pneumococcal infections, serotype, survival analysis.

\section{INTRODUCTION}

Invasive pneumococcal disease (IPD), caused by infection with Streptococcus pneumoniae, has a substantial global burden in young children [1] and adults [2].

\footnotetext{
* Author for correspondence: Dr R. Gorton, Public Health England North East, Citygate, Gallowgate, Newcastle upon Tyne, NE1 4WH, UK. (Email: russell.gorton@phe.gov.uk).
}

There are over 90 known serotypes of $S$. pneumoniae, differentiation of which is based on the composition of the polysaccharide capsule [3]. Different serotypes induce different immune responses and, together with other bacterial virulence factors and host risk factors, contribute to pathogenicity and severity of infection [3]. Given that vaccines against infection with S. pneumoniae are limited to certain subsets of serotypes, consideration of differential pathogenicity (and the 
resultant severity of infection and associated mortality) is important for maximizing the societal benefits of a vaccination programme [4].

There is a growing body of evidence to support the existence of serotype-specific mortality rates immediately following IPD [4-9]. Studies which include infection of children are limited in statistical power due to low mortality rates for this age group [4, 5], but there is clear evidence for adults that certain serotypes are associated with a relatively increased 30-day mortality compared to other serotypes [8]. In the longer-term, reduced life expectancy following pneumococcal pneumonia ( $\leqslant 10$ years) [10] and meningitis $(\leqslant 20$ years) [11] has also been observed. Generally, septicaemia is associated with increased rates of long-term mortality ( $\leqslant 6$ years), although it is not clear whether this is an independent consequence of pathology, or due to comorbidities or sequelae [12]. Whatever the mechanism, such an effect is likely for pneumococcal septicaemia.

Given the likely impact of IPD on longer-term mortality, it is important to understand whether serotype-specific associations with longer-term mortality exist. Such information may help to inform costeffectiveness studies for future vaccine formulations. Using enhanced surveillance data from the North East of England linked to registered deaths, we assessed both the short-term (30-day) and longer-term ( $\leqslant 7$ years) associations between individual serotypes of $S$. pneumoniae and mortality following diagnosis.

\section{METHODS}

\section{Study population}

The North East of England has a population of about 2.6 million persons [13], and has the least favourable self-reported general health in the country [14]. Life expectancy is among the lowest in the UK; 1.0 and $1 \cdot 1$ years below the national average for males and females, respectively [13]. On a small area level, the North East has a disproportionate level of social deprivation compared to other regions of the UK: $33 \%$ of small area populations of 1500 persons are within the most deprived national quintile [15].

\section{Surveillance data and linkage to mortality data}

Cases of IPD with a specimen date between 1 April 2006 and 31 March 2013 were obtained from the North East IPD enhanced surveillance system. Full details of the surveillance system are described elsewhere [16]. Briefly, all laboratories in the region notified local health protection staff of laboratoryconfirmed cases of IPD. Following notification, telephone interviews with laboratory staff and primary-/ secondary-care clinicians were conducted to obtain details of risk factors (indicators for pneumococcal vaccination agreed for England plus alcohol misuse) and vaccination status. Isolates were typed at the national reference laboratory. Cases were residents of the North East of England with S. pneumoniae detected from a normally sterile site. Age, sex, pneumococcal vaccination status, risk factors for IPD (alcohol misuse, chronic heart disease, chronic liver disease, chronic lung disease, chronic renal disease, diabetes, immunosuppression), and clinical presentation (bacteraemic pneumonia, meningitis, septicaemia) were routinely collected for each case. Other clinical presentations found at low frequency within the dataset (e.g. endocarditis, peritonitis) were grouped into a single category. Cases were attributed to quintiles of social deprivation as described elsewhere [17]. The social deprivation index used incorporates multiple domains (income, employment, health, education, housing, crime) to characterize small area living environments. All cases where the serotype was unknown were excluded. Serotypes which each accounted for $>1 \%$ of the dataset were considered individually. We limited analysis to cases without missing data for any variables and those which could be linked to mortality data. For cases where the positive specimen was taken on or after the date of death, specimen dates were adjusted to 1 day before the date of death. Cases were linked to Office for National Statistics registered deaths up to 30 September 2013 (data obtained March 2014) using unique National Health Service (NHS) numbers. Data was used in accordance with a data access agreement with Public Health England. The study was neither powered nor designed to test a specific hypothesis.

\section{Associations with mortality at 30 days, 12 months and 36 months post-diagnosis}

A binary logistic regression model was used to assess serotype-specific differences in all-cause mortality at three time points following diagnosis with IPD (30 days, 12 months, 36 months). Only cases with followup to the time point were included in that analysis. For each model, variables with a single variable association with outcome of $P<0 \cdot 2\left(\chi^{2}\right.$ for variables with two 
categories, Wald test for those with $>2$ ) were considered for inclusion in the multivariable model; age group, sex and serotype were retained irrespective of statistical significance. A backward selection model-building strategy was used, starting with a model containing all selected variables from the single variable analysis. Variables were then tested for exclusion if their coefficient in the model had an associated $P$ value $>0.05$ (starting with the variable with the highest $P$ value). Each reduced model was evaluated for fit using a likelihood ratio test. Goodness of fit for the final model (where all parameters had an associated $P$ value $<0.05$ ) was assessed using the Hosmer-Lemeshow goodness-of-fit test (with ten groups) [18] and a case classification table (cut-off $0 \cdot 5$ ). Marginal predicted probabilities of death at 30 days were obtained from the final multivariable model for each age group.

\section{Survival analysis}

A Cox proportional hazards $(\mathrm{PH})$ model was used to assess serotype-specific differences in all-cause mortality following diagnosis with IPD (defined as specimen date of the first positive test). An event was the day of death, truncated to 30 September 2013. To ensure acceptable precision at the end of the observation period, the period was limited to 7 years (truncating a small number of cases with data beyond this time point). Crude associations with mortality were assessed using the log rank test and any variables with an association of $P<0 \cdot 2$ were considered for inclusion in the Cox PH model. A backward selection model was used as for the logistic regression model. The assumption of PH of the final model was evaluated using a test of correlation of scaled Schoenfeld residuals with time. Where the $\mathrm{PH}$ assumption was not met $(P<0 \cdot 05)$, an extended Cox PH model was specified to include time-dependent effects (as a function of the natural logarithm of time) for each variable. The improvement of the extended model to the main-effects model was assessed using a likelihood ratio test. Adjusted Kaplan-Meier survival curves were used to assess serotype-specific effects after adjusting for all variables included in the $\mathrm{Cox} \mathrm{PH}$ model. We repeated the Cox PH model using an observation period starting 30 days after diagnosis.

\section{Statistical software}

All analysis was performed using Stata v. 13.1 (StataCorp., USA).

\section{RESULTS}

\section{Surveillance data and linkage to mortality data}

From the full surveillance dataset, 1779/1785 (99.7\%) IPD cases had NHS numbers available. Of these cases, $210(11 \cdot 8 \%)$ had no serotype data available. A further 253 cases $(16 \cdot 1 \%)$ had data missing for at least one variable (postcode, $n=26$; pneumococcal vaccination status, $n=84$; clinical presentation, $n=$ 22 ; $\geqslant 1$ risk factor, $n=162$ ) and were excluded; leaving 1316 cases for analysis $(74.0 \%$ of the original dataset, Table 1). Of this dataset, 24 serotypes each accounted for $>1 \%$ of cases ( $\geqslant 16$ cases per serotype) and were included as individual categories (Table 2). Forty-seven cases from this dataset were diagnosed with IPD on the date of death or at post-mortem; to enable inclusion of these cases in survival analysis, the specimen date was adjusted to the day before the date of death.

\section{Mortality at 30 days, 12 months and 36 months post-diagnosis}

Of the 1316 cases included in the analysis, a total of $243[18 \cdot 5 \%, 95 \%$ confidence interval (CI) 16.4-20.7] died within 30 days of diagnosis. As expected, mortality rates were higher when the follow-up period was extended to 12 months (364 deaths from the 1233 cases with sufficient follow-up time; $29 \cdot 5 \%, 95 \%$ CI 27.0-32.2) and 36 months (352 deaths from the 911 cases with sufficient follow-up time; $38 \cdot 6 \%, 95 \%$ CI 35.4-41.9). A similar pattern of increasing mortality rates with increasing age groups was seen for all three time points (Fig. 1).

A multivariable model for associations with mortality at 30 days contained age group, sex, serotype (retained irrespective of significance) plus deprivation, clinical presentation and number of risk factors (Supplementary Table S1, Table 3). The model has an overall acceptable goodness of fit $\left(\chi^{2}=8 \cdot 46\right.$, D.F. $=$ $8, P=0 \cdot 390)$, no reduction in fit compared to the starting model $\left(\chi^{2}=6 \cdot 30\right.$, D.F. $\left.=7, P=0.505\right)$ and correct classification of $82 \%$ of cases. From this model, four serotypes $(3,6 \mathrm{~A}, 9 \mathrm{~N}, 19 \mathrm{~F})$ were significantly associated with an overall increased mortality compared to the group of other serotypes. There is little variation in predicted probabilities of mortality at 30 days by serotype in children and younger adults (Fig. 2). Those serotypes found to have an overall significant association with increased mortality at 30 days are only predicted to have a rate significantly 
Table 1. Characteristics of IPD cases

\begin{tabular}{|c|c|c|c|c|}
\hline \multirow[b]{2}{*}{ Variable } & \multirow[b]{2}{*}{ Category* } & \multicolumn{3}{|c|}{ No. of cases $(\%)$} \\
\hline & & In category & Yes & No \\
\hline \multirow[t]{5}{*}{ Age group, years } & $0-19$ & $195(14 \cdot 8)$ & - & - \\
\hline & $20-39$ & $137(10 \cdot 4)$ & - & - \\
\hline & $40-59$ & $275(20 \cdot 9)$ & - & - \\
\hline & $60-79$ & $446(33.9)$ & - & - \\
\hline & $\geqslant 80$ & $263(20 \cdot 0)$ & & \\
\hline \multirow[t]{2}{*}{ Sex } & Male & $686(52 \cdot 1)$ & - & - \\
\hline & Female & $630(47 \cdot 9)$ & - & - \\
\hline \multirow[t]{7}{*}{ Year of diagnosis } & 2006-2007 & $209(15 \cdot 9)$ & - & - \\
\hline & $2007-2008$ & $235(17 \cdot 9)$ & - & - \\
\hline & 2008-2009 & $188(14 \cdot 3)$ & - & - \\
\hline & 2009-2010 & $208(15 \cdot 8)$ & - & - \\
\hline & 2010-2011 & $203(15 \cdot 4)$ & - & - \\
\hline & 2011-2012 & $150(11 \cdot 4)$ & - & - \\
\hline & 2012-2013 & $123(9 \cdot 4)$ & - & - \\
\hline \multirow{5}{*}{ Deprivation quintile $\dagger$} & Quintile 1 & $186(14 \cdot 1)$ & - & - \\
\hline & Quintile 2 & $241(18 \cdot 3)$ & - & - \\
\hline & Quintile 3 & $261(19 \cdot 8)$ & - & - \\
\hline & Quintile 4 & $284(21 \cdot 6)$ & - & - \\
\hline & Quintile 5 & $344(26 \cdot 1)$ & - & - \\
\hline Pneumococcal immunization & Vaccinated & - & $604(45 \cdot 9)$ & $712(54 \cdot 1)$ \\
\hline \multirow[t]{4}{*}{ Clinical presentation } & Meningitis & $125(9 \cdot 5)$ & - & - \\
\hline & Pneumonia & $908(69 \cdot 0)$ & - & - \\
\hline & Septicaemia & $125(9 \cdot 5)$ & - & - \\
\hline & Other & $158(12 \cdot 0)$ & - & - \\
\hline Alcohol misuse & - & - & $113(8 \cdot 6)$ & $1203(91 \cdot 4)$ \\
\hline Chronic heart disease & - & - & $291(22 \cdot 1)$ & $1025(77 \cdot 9)$ \\
\hline Chronic liver disease & - & - & $58(4 \cdot 4)$ & $1258(95 \cdot 6)$ \\
\hline Chronic lung disease & - & - & $323(24 \cdot 5)$ & $993(75 \cdot 5)$ \\
\hline Chronic renal disease & - & - & $141(10 \cdot 7)$ & $1175(89 \cdot 3)$ \\
\hline Diabetes & - & - & $151(11 \cdot 5)$ & $1165(88 \cdot 5)$ \\
\hline Immunosuppression & - & - & $145(11 \cdot 0)$ & $1171(89 \cdot 0)$ \\
\hline \multirow{3}{*}{ Number of IPD risk factors } & 0 & $610(46 \cdot 4)$ & - & - \\
\hline & 1 & $396(30 \cdot 1)$ & - & - \\
\hline & $\geqslant 2$ & $310(23 \cdot 6)$ & - & - \\
\hline
\end{tabular}

IPD, Invasive pneumococcal disease.

* Missing category only presented where $\geqslant 1$ case had missing data for that variable.

$\dagger$ Quintile 1, least deprived; quintile 5, most deprived.

$\vdots$ Includes alcohol misuse, chronic heart disease, chronic liver disease, chronic lung disease, chronic renal disease, diabetes, immunosuppression.

above the age group average for older adults $(\geqslant 60$ years).

After extending the all-cause mortality window to 12 months, one serotype remained associated with increased odds of mortality by this time point (19 F) and one serotype (1) was associated with reduced odds of mortality, both compared to the group of other serotypes (Table 3). Nonetheless, there remained substantial variation in predicted 12-month all-cause mortality rates with different serotypes for older age groups (Fig. 3). When comparing significant predictors to the 30-day all-cause mortality model, deprivation and clinical presentation were no longer significant predictors of death, while immunosuppression was now associated of increased odds of death and chronic renal disease was associated with reduced odds of death (Supplementary Table S2, Table 3). The 12-month mortality model also showed an acceptable goodness of fit $\left(\chi^{2}=7 \cdot 49\right.$, D.F. $\left.=8, P=0 \cdot 485\right)$, no reduction in fit compared to the full starting model $\left(\chi^{2}=5 \cdot 17\right.$, D.F. $\left.=7, P=0 \cdot 640\right)$, and a good classification rate for cases $(77 \%)$. 
Table 2. Distribution of serotypes of invasive pneumococcal disease cases

\begin{tabular}{lcr}
\hline \hline Serotype* & No. of cases & $\%$ \\
\hline 1 & 165 & $12 \cdot 54$ \\
3 & 114 & $8 \cdot 66$ \\
4 & 35 & $2 \cdot 66$ \\
$6 \mathrm{~A}$ & 34 & $2 \cdot 58$ \\
$6 \mathrm{~B}$ & 26 & $1 \cdot 98$ \\
$6 \mathrm{C}$ & 33 & $2 \cdot 51$ \\
$7 \mathrm{~F}$ & 150 & $11 \cdot 40$ \\
8 & 98 & $7 \cdot 45$ \\
$9 \mathrm{~N}$ & 22 & $1 \cdot 67$ \\
$9 \mathrm{~V}$ & 31 & $2 \cdot 36$ \\
$10 \mathrm{~A}$ & 16 & $1 \cdot 22$ \\
$11 \mathrm{~A}$ & 22 & $1 \cdot 67$ \\
$12 \mathrm{~F}$ & 38 & $2 \cdot 89$ \\
14 & 41 & $3 \cdot 12$ \\
$18 \mathrm{C}$ & 24 & $1 \cdot 82$ \\
$19 \mathrm{~A}$ & 117 & $8 \cdot 89$ \\
$19 \mathrm{~F}$ & 24 & $1 \cdot 82$ \\
20 & 16 & $1 \cdot 22$ \\
$22 \mathrm{~F}$ & 90 & $6 \cdot 84$ \\
$23 \mathrm{~A}$ & 21 & $1 \cdot 60$ \\
$23 \mathrm{~F}$ & 35 & $2 \cdot 66$ \\
31 & 16 & $1 \cdot 22$ \\
$33 \mathrm{~F}$ & 34 & $2 \cdot 58$ \\
$35 \mathrm{~F}$ & 16 & $1 \cdot 22$ \\
Other serotypes $\dagger$ & 98 & $7 \cdot 45$ \\
\hline \hline
\end{tabular}

* Serotypes which each account for $>1 \%$ of the dataset.

$\dagger$ Serotypes 2, 5, 7, 9A, 10F, 12B, 15A, 15B, 15C, 16, 16A, $16 \mathrm{~F}, 17 \mathrm{~F}, 19,21,23,23 \mathrm{~B}, 24 \mathrm{~F}, 25 \mathrm{~F}, 28 \mathrm{~A}, 29,33 \mathrm{~A}, 34$, $35 \mathrm{~B}, 38$.

Extending the mortality window to 36 months further reduced the association of serotype with mortality (Table 3). Only one individual serotype (1) remained a significant predictor of (a reduced) all-cause mortality at 36 months compared to the group of other serotypes. For this model, the number of IPD risk factors was no longer a significant predictor, but three additional individual risk factors (chronic heart disease, chronic liver disease, chronic lung disease, plus immunosuppression also included in the 12-month model) were now associated with increased odds of death (Supplementary Table S3, Table 3). Similarly to the 30-day model (but not the 12-month model), deprivation was a significant predictor of all-cause mortality within 36 months. As with both other time-point models there was substantial variation in predicted all-cause mortality rates with different serotypes for older age groups (Fig. 4). The 36-month mortality model also showed an acceptable goodness of fit $\left(\chi^{2}=5 \cdot 20\right.$, D.F. $\left.=8, P=0 \cdot 736\right)$, no reduction in fit compared to the full starting model $\chi^{2}=5 \cdot 07$, D.F. $=7, P=0.651)$, and a good classification rate for cases $(78 \%)$.

Sensitivity analysis of all-cause mortality by time points showed no pattern or substantial change in crude odds ratios when using all available cases and not just cases without missing data: 30-day (median $+18 \%$ change, range $-119 \%$ to $+26 \%$ ); 12 months (median $-8 \%$ change, range $-80 \%$ to $+99 \%$ ); 36 months (median $-10 \%$ change, range $-62 \%$ to $+9 \%$ ). Given the small numbers of cases for certain serotypes, changes in the significance of associations were found, although there was no trend associated with the sensitivity analysis (Supplementary Table S4). Using all available data for final multivariable models did not lead to the loss of any significant associations for individual serotypes (Supplementary Table S5).

\section{Analysis of longer-term survival}

Of the 1316 cases included in the analysis, $542(41 \cdot 2 \%$, 95\% CI 38.5-43.9) died within the available follow-up period (from date of diagnosis to 30 September 2013; median 889 days, range 1-2557). For the all cases model, the main-effects Cox PH model included age group, sex, deprivation, serotype, clinical presentation, chronic heart disease, chronic liver disease, chronic lung disease, and immunosuppression (Supplementary Table S6, Table 4). Sensitivity analysis using all available cases rather than just cases without any missing data suggested no substantial change in crude associations if all available data were included (Supplementary Table S4). The assumption of $\mathrm{PH}$ was not met for three age groups (40-59, 60-79, $\geqslant 80$ years), sex, one serotype (3), one clinical presentation (meningitis) and immunosuppression. Subsequent time-dependent effects were significant $(P<0 \cdot 05)$ for all three age groups, sex, clinical presentation (meningitis) and immunosuppression (Supplementary Table S6). The extended Cox PH model represented an overall significantly improved fit compared to the main-effects model $\chi^{2}=55.99$, D.F. $=6, P<0.001)$ and the results of this model were used for assessment of the hazard ratio (HR) (Table 4). Two serotypes (1, reduced mortality; 9N, increased mortality) had statistically significant HRs, demonstrating serotype-specific variation in survival following infection, after adjustment for other significant predictors. Those average serotypespecific effects are restricted to older adults (Fig. 5). 


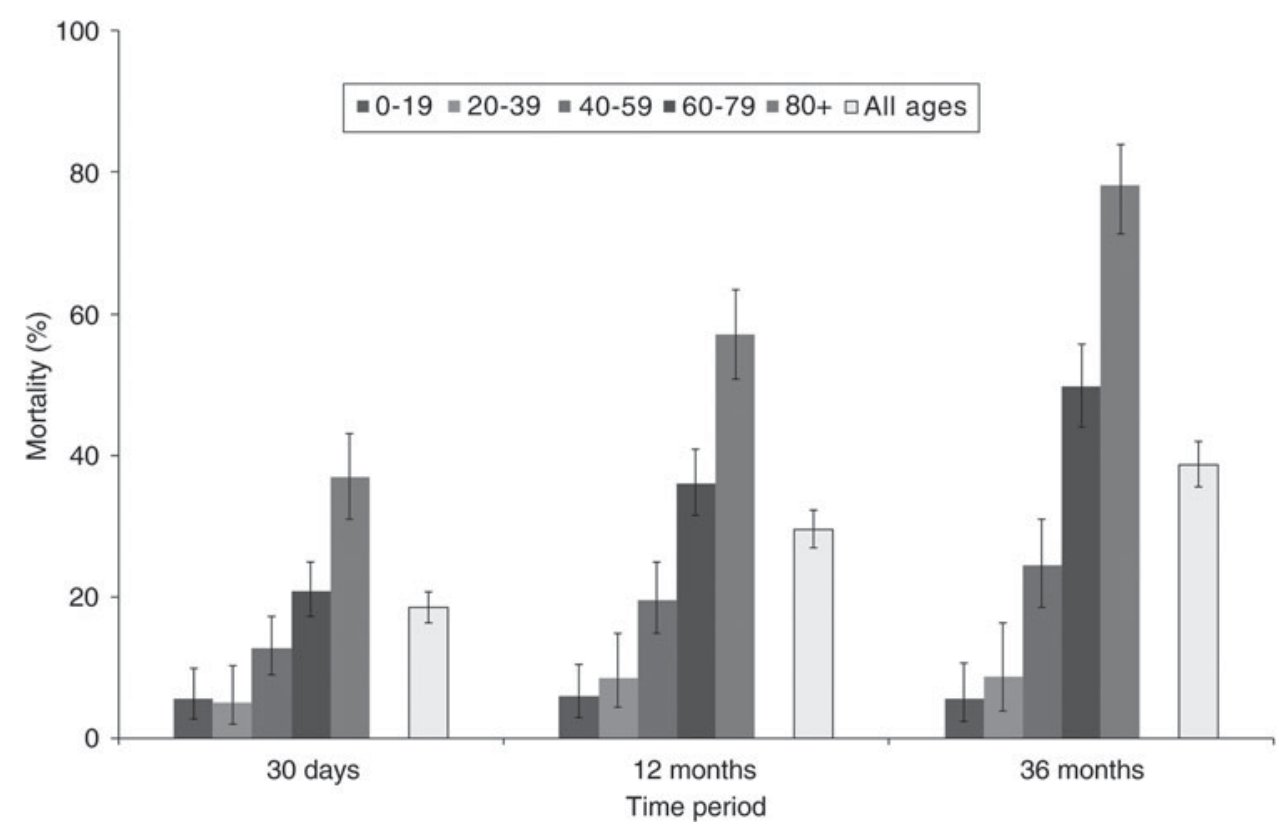

Fig. 1. Crude mortality rates at 30 days, 12 months and 36 months post-diagnosis with invasive pneumococcal disease by age groups.

There was no change in the significance of maineffects predictors for serotypes using all available data for the final multivariable model (Supplementary Table S5).

The analysis using an observation period starting 30 days after diagnosis included 1073 cases, of which $299(27 \cdot 9 \%, 95 \%$ CI $25 \cdot 2-30 \cdot 7)$ died within the available follow-up period (median 1140 days, range 1-2527). The Cox PH model included main effects for age group, sex, serotype, clinical presentation, chronic heart disease, chronic liver disease, chronic lung disease and immunosuppression (Supplementary Table S5, Table 4). The assumption of $\mathrm{PH}$ was not met for two serotypes $(1,35 \mathrm{~F})$ and chronic liver disease. Subsequent time-dependent effects were significant $(P<0.05)$ only for serotype $35 \mathrm{~F}$ (Supplementary Table S5). The extended Cox $\mathrm{PH}$ model represented an overall significantly improved fit compared to the main-effects model $\left(\chi^{2}=4 \cdot 81\right.$, D.F. $\left.=1, P=0 \cdot 028\right)$ and the results of this model were used for assessment of the HR (Table 4). Six serotypes (1, 7 F, 19A, $22 \mathrm{~F}, 23 \mathrm{~F}$, $35 \mathrm{~F})$ had statistically significant HRs, although only one of these $(35 \mathrm{~F})$ was for an increased risk of all-cause mortality. The association between serotype $9 \mathrm{~N}$ and increased all-cause mortality found in the model containing the whole observation period (Table 3) was not found when excluding the first 30 days following diagnosis from the observation period (and thus removing from analysis the 243 cases that died within that time period).

\section{DISCUSSION}

Given the requirement to demonstrate the costeffectiveness of vaccination programmes, together with the need to select only certain serotypes for inclusion in vaccine formulations, the existence of serotypespecific mortality has implications for determining the societal benefits of pneumococcal vaccines. It is wellestablished that mortality rates immediately following IPD are associated with the serotype of $S$. pneumoniae causing the infection [8]. This is, to our knowledge, the first study to consider the potential association between serotype and longer-term mortality. Using a cohort of IPD cases from a region of England with a population of $\sim 2.5$ million persons, we have estimated the relative associations between short-term (30-day) and long-term ( $\leqslant 7$ years) all-cause mortality following IPD and 24 different serotypes of S. pneumoniae.

Our findings support those of previous studies that pneumococcal serotype is independently associated with differential 30-day mortality rates following IPD. After adjusting for confounding by the effects of the living environment (deprivation), comorbidities, and clinical presentation, four serotypes were associated with increased mortality rates at 30 days $(3,6 \mathrm{~A}, 9 \mathrm{~N}, 19 \mathrm{~F})$. These associations are supported 
Table 3. Crude and adjusted associations with mortality at 30 days, 12 months and 36 months post-diagnosis with invasive pneumococcal disease

\begin{tabular}{|c|c|c|c|c|c|c|c|c|c|c|c|c|c|}
\hline \multirow[b]{3}{*}{ Variable } & \multirow[b]{3}{*}{ Category } & \multicolumn{4}{|l|}{ 30-day survival } & \multicolumn{4}{|l|}{ 12-month survival } & \multicolumn{4}{|l|}{ 36-month survival } \\
\hline & & \multicolumn{2}{|c|}{ Single variable analysis } & \multicolumn{2}{|c|}{ Multivariable analysis } & \multicolumn{2}{|c|}{ Single variable analysis } & \multicolumn{2}{|c|}{ Multivariable analysis } & \multicolumn{2}{|l|}{ Single variable analysis } & \multicolumn{2}{|c|}{ Multivariable analysis } \\
\hline & & OR $(95 \% \mathrm{CI})$ & $P^{*}$ & aOR $(95 \%$ CI $)$ & $P$ & OR $(95 \% \mathrm{CI})$ & $P^{*}$ & aOR $(95 \% \mathrm{CI})$ & $P$ & OR $(95 \% \mathrm{CI})$ & $P^{*}$ & aOR $(95 \%$ CI $)$ & $P$ \\
\hline \multirow{5}{*}{$\begin{array}{l}\text { Age group, } \\
\text { years } \dagger\end{array}$} & $0-19$ & Ref. & $<0.001$ & Ref. & - & Ref. & $<0 \cdot 001$ & Ref. & - & Ref. & $<0 \cdot 001$ & Ref. & - \\
\hline & $20-39$ & $0 \cdot 90(0 \cdot 34-2 \cdot 39)$ & 0.833 & $0 \cdot 98(0 \cdot 36-2 \cdot 67)$ & 0.966 & $1.48(0.62-3.52)$ & $0 \cdot 377$ & $1 \cdot 77(0 \cdot 72-4 \cdot 37)$ & $0 \cdot 215$ & $1 \cdot 61(0 \cdot 58-4 \cdot 44)$ & $0 \cdot 361$ & $1 \cdot 73(0.59-5.07)$ & $0 \cdot 317$ \\
\hline & $40-59$ & $2.44(1.21-4.93)$ & 0.013 & $2 \cdot 04(0 \cdot 97-4 \cdot 28)$ & $0 \cdot 060$ & $3.83(1.94-7.60)$ & $<0.001$ & $3.09(1.50-6.35)$ & 0.002 & $5.44(2.49-11.90)$ & $<0.001$ & $4 \cdot 17(1 \cdot 81-9 \cdot 62)$ & $<0.001$ \\
\hline & $60-79$ & $4 \cdot 40(2 \cdot 30-8 \cdot 44)$ & $<0.001$ & $2.96(1.46-5.98)$ & $0 \cdot 003$ & $8 \cdot 89(4 \cdot 68-16 \cdot 87)$ & $<0.001$ & $6 \cdot 16(3 \cdot 11-12 \cdot 20)$ & $<0.001$ & $16 \cdot 76(7 \cdot 93-35 \cdot 43)$ & $<0.001$ & $12 \cdot 30(5 \cdot 55-27 \cdot 26)$ & $<0.001$ \\
\hline & $\geqslant 80$ & $9 \cdot 77(5 \cdot 06-18 \cdot 87)$ & $<0 \cdot 001$ & $6 \cdot 58(3 \cdot 20-13 \cdot 50)$ & $<0.001$ & $20 \cdot 77(10 \cdot 74-40 \cdot 17)$ & $<0 \cdot 001$ & $17 \cdot 76(8 \cdot 71-36 \cdot 19)$ & $<0.001$ & $60 \cdot 14(27 \cdot 11-133 \cdot 42)$ & $<0 \cdot 001$ & $62 \cdot 86(26 \cdot 54-148 \cdot 90)$ & $<0.001$ \\
\hline \multirow{2}{*}{$\operatorname{Sex} \dagger$} & Female & Ref. & - & Ref. & - & Ref. & & Ref. & - & Ref. & - & Ref. & - \\
\hline & Male & $1 \cdot 07(0 \cdot 81-1 \cdot 41)$ & 0.636 & $1.28(0 \cdot 94-1 \cdot 75)$ & $0 \cdot 116$ & $1 \cdot 14(0 \cdot 89-1 \cdot 45)$ & $0 \cdot 303$ & $1.64(1 \cdot 23-2 \cdot 45)$ & 0.001 & $1 \cdot 17(0 \cdot 89-1 \cdot 53)$ & $0 \cdot 244$ & $1 \cdot 96(1 \cdot 37-2 \cdot 81)$ & $<0.001$ \\
\hline \multirow{7}{*}{$\begin{array}{l}\text { Year of } \\
\text { diagnosis }\end{array}$} & 2006-2007 & Ref. & 0.542 & - & - & Ref. & $0 \cdot 447$ & - & - & Ref. & $0 \cdot 460$ & - & - \\
\hline & $2007-2008$ & $0 \cdot 94(0 \cdot 59-1 \cdot 23)$ & $0 \cdot 775$ & - & - & $0 \cdot 83(0 \cdot 55-1 \cdot 23)$ & $0 \cdot 353$ & - & - & $1.06(0.73-1.56)$ & $0 \cdot 747$ & - & - \\
\hline & 2008-2009 & $0 \cdot 64(0 \cdot 38-1 \cdot 07)$ & 0.090 & - & - & $0 \cdot 72(0 \cdot 46-1 \cdot 10)$ & $0 \cdot 131$ & - & - & $0 \cdot 75(0 \cdot 50-1 \cdot 13)$ & $0 \cdot 169$ & - & - \\
\hline & 2009-2010 & $0.89(0.56-1.44)$ & $0 \cdot 646$ & - & - & $0 \cdot 78(0.52-1 \cdot 19)$ & $0 \cdot 256$ & - & - & $0.97(0 \cdot 65-1 \cdot 43)$ & $0 \cdot 873$ & - & - \\
\hline & 2010-2011 & $0 \cdot 81(0 \cdot 50-1 \cdot 32)$ & 0.326 & - & - & $0 \cdot 74(0 \cdot 48-1 \cdot 12)$ & $0 \cdot 156$ & - & - & $0 \cdot 81(0 \cdot 46-1 \cdot 42)$ & $0 \cdot 458$ & - & - \\
\hline & 2011-2012 & $0 \cdot 76(0 \cdot 45-1 \cdot 31)$ & $0 \cdot 195$ & - & - & $1 \cdot 11(0 \cdot 71-1 \cdot 72)$ & $0 \cdot 647$ & - & - & - & - & - & - \\
\hline & 2012-2013 & $0 \cdot 62(0 \cdot 34-1 \cdot 14)$ & $0 \cdot 124$ & - & - & $0.98(0.47-2 \cdot 01)$ & 0.949 & - & - & - & - & - & - \\
\hline \multirow{5}{*}{ Deprivation } & Quintile 1 & Ref & 0.050 & Ref. & - & Ref. & $0 \cdot 486$ & - & - & Ref. & 0.065 & Ref. & - \\
\hline & Quintile 2 & $1 \cdot 26(0 \cdot 74-2 \cdot 15)$ & 0.393 & $1.33(0 \cdot 75-2 \cdot 37)$ & 0.336 & $1.21(0.78-1.89)$ & $0 \cdot 391$ & - & - & $1.06(0.64-1 \cdot 75)$ & $0 \cdot 817$ & $0.85(0.44-1.63)$ & $0 \cdot 621$ \\
\hline & Quintile 3 & $1 \cdot 39(0 \cdot 82-2 \cdot 33)$ & $0 \cdot 217$ & $1 \cdot 50(0 \cdot 85-2 \cdot 64)$ & $0 \cdot 165$ & $1 \cdot 12(0 \cdot 72-1 \cdot 73)$ & $0 \cdot 612$ & - & - & $1 \cdot 50(0 \cdot 93-2 \cdot 43)$ & 0.099 & $1 \cdot 60(0 \cdot 85-3 \cdot 00)$ & $0 \cdot 144$ \\
\hline & Quintile 4 & $1.97(1.20-3.24)$ & 0.007 & $2 \cdot 30(1 \cdot 34-3 \cdot 95)$ & $0 \cdot 003$ & $1 \cdot 41(0 \cdot 92-2 \cdot 15)$ & $0 \cdot 113$ & - & - & $1 \cdot 75(1 \cdot 10-2 \cdot 80)$ & $0 \cdot 019$ & $2 \cdot 18(1 \cdot 18-4 \cdot 01)$ & $0 \cdot 012$ \\
\hline & Quintile 5 & $1 \cdot 27(0 \cdot 77-2 \cdot 10)$ & $0 \cdot 343$ & $1 \cdot 50(0 \cdot 87-2 \cdot 60)$ & 0.647 & $1.08(0.71-1.63)$ & $0 \cdot 724$ & - & - & $1 \cdot 24(0 \cdot 78-1 \cdot 97)$ & $0 \cdot 360$ & $1 \cdot 47(0 \cdot 80-2 \cdot 68)$ & $0 \cdot 213$ \\
\hline \multirow{2}{*}{ Immunization } & Unvaccinated & Ref. & - & - & - & Ref. & - & - & - & Ref. & - & - & - \\
\hline & Vaccinated & $1.85(1.36-2.52)$ & $<0 \cdot 001$ & - & - & $2 \cdot 17(1 \cdot 69-2 \cdot 79)$ & $<0 \cdot 001$ & - & - & $2 \cdot 77(2 \cdot 11-3 \cdot 65)$ & $<0 \cdot 001$ & - & - \\
\hline \multirow[t]{20}{*}{ Serotype } & Other $†$ & Ref. & $<0 \cdot 001$ & Ref. & - & Ref. & $<0 \cdot 001$ & - & - & Ref. & $<0 \cdot 001$ & Ref. & - \\
\hline & 1 & $0 \cdot 38(0 \cdot 16-0 \cdot 90)$ & $0 \cdot 028$ & $0 \cdot 81(0.33-1.99)$ & 0.643 & $0 \cdot 16(0 \cdot 08-0 \cdot 34)$ & $<0.001$ & $0 \cdot 30(0 \cdot 13-0 \cdot 66)$ & $0 \cdot 003$ & $0 \cdot 17(0 \cdot 08-0 \cdot 36)$ & $<0 \cdot 001$ & $0 \cdot 33(0 \cdot 13-0 \cdot 80)$ & $0 \cdot 015$ \\
\hline & 3 & $2 \cdot 24(1 \cdot 11-4 \cdot 51)$ & 0.024 & $2 \cdot 20(1 \cdot 05-4 \cdot 60)$ & 0.036 & $1.32(0.73-2 \cdot 38)$ & $0 \cdot 352$ & $1 \cdot 22(0 \cdot 64-2 \cdot 34)$ & 0.549 & $1 \cdot 05(0 \cdot 51-2 \cdot 16)$ & $0 \cdot 890$ & $0.89(0.37-2 \cdot 18)$ & $0 \cdot 802$ \\
\hline & 4 & $1 \cdot 00(0 \cdot 33-3 \cdot 01)$ & $1 \cdot 000$ & $1.22(0 \cdot 38-3.94)$ & $0 \cdot 742$ & $0 \cdot 41(0 \cdot 15-1 \cdot 11)$ & $0 \cdot 079$ & $0 \cdot 43(0 \cdot 15-1 \cdot 26)$ & $0 \cdot 124$ & $0 \cdot 39(0 \cdot 15-1 \cdot 04)$ & $0 \cdot 060$ & $0 \cdot 31(0 \cdot 09-1 \cdot 01)$ & $0 \cdot 051$ \\
\hline & $6 \mathrm{~A}$ & $3 \cdot 27(1 \cdot 33-8 \cdot 07)$ & $0 \cdot 010$ & $3 \cdot 11(1 \cdot 19-8 \cdot 17)$ & 0.021 & $2 \cdot 40(1 \cdot 06-5 \cdot 43)$ & $0 \cdot 036$ & $2 \cdot 10(0 \cdot 84-5 \cdot 25)$ & $0 \cdot 113$ & $1 \cdot 70(0 \cdot 68-4 \cdot 22)$ & $0 \cdot 253$ & $1 \cdot 42(0 \cdot 47-4 \cdot 31)$ & 0.533 \\
\hline & $6 \mathrm{~B}$ & $2 \cdot 21(0 \cdot 79-6 \cdot 22)$ & $0 \cdot 133$ & $2 \cdot 12(0 \cdot 71-6 \cdot 30)$ & $0 \cdot 177$ & $2.00(0.82-4 \cdot 86)$ & $0 \cdot 126$ & $1.63(0.62-4.29)$ & $0 \cdot 327$ & $1.56(0 \cdot 59-4 \cdot 12)$ & $0 \cdot 367$ & $1 \cdot 16(0 \cdot 37-3 \cdot 60)$ & 0.796 \\
\hline & $6 \mathrm{C}$ & $3.00(1.20-7.52)$ & $0 \cdot 019$ & $2 \cdot 33(0 \cdot 88-6 \cdot 20)$ & $0 \cdot 089$ & $2 \cdot 29(1.09-5.99)$ & 0.032 & $1 \cdot 70(0 \cdot 68-4 \cdot 22)$ & $0 \cdot 249$ & $1 \cdot 22(0 \cdot 35-4 \cdot 34)$ & 0.751 & $0 \cdot 89(0 \cdot 20-4 \cdot 01)$ & $0 \cdot 884$ \\
\hline & $7 \mathrm{~F}$ & $0 \cdot 77(0 \cdot 36-1 \cdot 64)$ & 0.493 & $1 \cdot 12(0 \cdot 51-2 \cdot 49)$ & 0.776 & $0 \cdot 46(0 \cdot 24-0 \cdot 86)$ & $0 \cdot 015$ & $0 \cdot 62(0 \cdot 31-1 \cdot 23)$ & $0 \cdot 168$ & $0 \cdot 50(0 \cdot 24-1 \cdot 03)$ & $0 \cdot 060$ & $0 \cdot 66(0 \cdot 27-1 \cdot 58)$ & $0 \cdot 347$ \\
\hline & 8 & $0 \cdot 76(0 \cdot 33-1 \cdot 77)$ & 0.522 & $0 \cdot 83(0 \cdot 35-2 \cdot 00)$ & $0 \cdot 680$ & $0 \cdot 63(0 \cdot 32-1 \cdot 16)$ & $0 \cdot 185$ & $0 \cdot 54(0 \cdot 26-1 \cdot 12)$ & $0 \cdot 096$ & $0 \cdot 72(0 \cdot 34-1 \cdot 55)$ & $0 \cdot 406$ & $0 \cdot 47(0 \cdot 19-1 \cdot 17)$ & $0 \cdot 104$ \\
\hline & $9 \mathrm{~N}$ & $4 \cdot 15(1 \cdot 50-11 \cdot 53)$ & $0 \cdot 006$ & $4 \cdot 48(1 \cdot 50-13 \cdot 37)$ & 0.007 & $2 \cdot 40(0 \cdot 93-6 \cdot 21)$ & $0 \cdot 071$ & $2 \cdot 35(0 \cdot 82-6 \cdot 70)$ & $0 \cdot 110$ & $2 \cdot 45(0 \cdot 79-7 \cdot 60)$ & $0 \cdot 119$ & $2 \cdot 56(0 \cdot 65-10 \cdot 20)$ & $0 \cdot 181$ \\
\hline & $9 \mathrm{~V}$ & $1 \cdot 75(0 \cdot 63-4 \cdot 83)$ & $0 \cdot 280$ & $1 \cdot 50(0 \cdot 52-4 \cdot 35)$ & 0.453 & $0 \cdot 95(0 \cdot 40-2 \cdot 28)$ & $0 \cdot 913$ & $0 \cdot 63(0 \cdot 24-1 \cdot 64)$ & $0 \cdot 348$ & $0 \cdot 87(0 \cdot 34-2 \cdot 19)$ & 0.762 & $0 \cdot 39(0 \cdot 13-1 \cdot 16)$ & $0 \cdot 089$ \\
\hline & $10 \mathrm{~A}$ & $2 \cdot 00(0 \cdot 56-7 \cdot 09)$ & $0 \cdot 283$ & $2 \cdot 63(0 \cdot 66-10 \cdot 42)$ & $0 \cdot 169$ & $0 \cdot 55(0 \cdot 14-2 \cdot 11)$ & $0 \cdot 380$ & $0 \cdot 61(0 \cdot 13-2 \cdot 81)$ & 0.529 & - & - & - & - \\
\hline & $11 \mathrm{~A}$ & $3 \cdot 43(1 \cdot 22-9 \cdot 67)$ & 0.020 & $2 \cdot 66(0 \cdot 90-7 \cdot 91)$ & 0.078 & $2 \cdot 89(1 \cdot 11-7 \cdot 54)$ & $\mathbf{0 . 0 3 0}$ & $2 \cdot 39(0 \cdot 84-6 \cdot 80)$ & $0 \cdot 103$ & $4.09(1 \cdot 00-16 \cdot 72)$ & $0 \cdot 050$ & $2 \cdot 76(0 \cdot 55-13 \cdot 89)$ & $0 \cdot 219$ \\
\hline & $12 \mathrm{~F}$ & $0 \cdot 91(0 \cdot 30-2 \cdot 72)$ & $0 \cdot 865$ & $0.93(0.30-2.91)$ & $0 \cdot 900$ & $0.85(0.37-1.95)$ & $0 \cdot 695$ & $0 \cdot 80(0 \cdot 32-1.98)$ & $0 \cdot 625$ & $0 \cdot 47(0 \cdot 17-1 \cdot 26)$ & $0 \cdot 132$ & $0 \cdot 42(0 \cdot 13-1 \cdot 32)$ & $0 \cdot 138$ \\
\hline & 14 & $1 \cdot 24(0 \cdot 46-3 \cdot 33)$ & 0.676 & $1 \cdot 12(0 \cdot 40-3 \cdot 18)$ & 0.825 & $1 \cdot 20(0.55-2 \cdot 62)$ & $0 \cdot 647$ & $0.67(0.27-1.62)$ & $0 \cdot 370$ & $1 \cdot 23(0 \cdot 52-2 \cdot 87)$ & 0.636 & $0 \cdot 64(0 \cdot 22-1 \cdot 90)$ & $0 \cdot 427$ \\
\hline & $18 \mathrm{C}$ & $0 \cdot 86(0 \cdot 23-3 \cdot 26)$ & 0.821 & $0 \cdot 79(0 \cdot 19-3 \cdot 22)$ & 0.738 & $0 \cdot 67(0 \cdot 24-1 \cdot 86)$ & 0.439 & $0 \cdot 61(0 \cdot 20-1 \cdot 90)$ & 0.399 & $0 \cdot 70(0 \cdot 25-1 \cdot 97)$ & 0.502 & $0 \cdot 68(0 \cdot 19-2 \cdot 44)$ & 0.555 \\
\hline & $19 \mathrm{~A}$ & $1 \cdot 63(0 \cdot 80-3 \cdot 34)$ & $0 \cdot 182$ & $1.55(0.72-3.32)$ & 0.259 & $0 \cdot 67(0 \cdot 36-1 \cdot 24)$ & $0 \cdot 198$ & $0.52(0.26-1.02)$ & 0.058 & $0.74(0.35-1.57)$ & $0 \cdot 428$ & $0 \cdot 48(0 \cdot 19-1 \cdot 24)$ & $0 \cdot 129$ \\
\hline & $19 \mathrm{~F}$ & $3.60(1.32-9.80)$ & 0.012 & $3.90(1.32-11.49)$ & 0.013 & $2 \cdot 80(1 \cdot 11-7 \cdot 07)$ & 0.029 & $3.27(1 \cdot 13-9 \cdot 43)$ & 0.029 & $1 \cdot 84(0 \cdot 64-5 \cdot 30)$ & $0 \cdot 258$ & $2 \cdot 27(0 \cdot 64-8 \cdot 51)$ & $0 \cdot 225$ \\
\hline & 20 & $1 \cdot 38(0 \cdot 35-5 \cdot 49)$ & 0.643 & $1 \cdot 20(0 \cdot 28-5 \cdot 07)$ & $0 \cdot 804$ & $0 \cdot 67(0 \cdot 20-2 \cdot 25)$ & $0 \cdot 396$ & $0 \cdot 51(0 \cdot 14-1 \cdot 84)$ & $0 \cdot 300$ & $1 \cdot 02(0 \cdot 27-3 \cdot 80)$ & 0.973 & $0 \cdot 63(0 \cdot 14-2 \cdot 80)$ & $0 \cdot 544$ \\
\hline & $22 \mathrm{~F}$ & $1 \cdot 40(0 \cdot 35-5 \cdot 46)$ & $0 \cdot 397$ & $1.26(0 \cdot 56-2.83)$ & 0.575 & $0 \cdot 74(0.39-1.43)$ & $0 \cdot 371$ & $0 \cdot 56(0 \cdot 27-1 \cdot 16)$ & $0 \cdot 120$ & $0 \cdot 96(0 \cdot 44-2 \cdot 07)$ & 0.915 & $0 \cdot 86(0 \cdot 34-2 \cdot 19)$ & $0 \cdot 749$ \\
\hline
\end{tabular}


Table 3 (cont.)

\begin{tabular}{|c|c|c|c|c|c|c|c|c|c|c|c|c|c|}
\hline \multirow[b]{3}{*}{ Variable } & \multirow[b]{3}{*}{ Category } & \multicolumn{4}{|l|}{ 30-day survival } & \multicolumn{4}{|l|}{ 12-month survival } & \multicolumn{4}{|l|}{ 36-month survival } \\
\hline & & \multicolumn{2}{|c|}{ Single variable analysis } & \multicolumn{2}{|c|}{ Multivariable analysis } & \multicolumn{2}{|c|}{ Single variable analysis } & \multicolumn{2}{|c|}{ Multivariable analysis } & \multicolumn{2}{|c|}{ Single variable analysis } & \multicolumn{2}{|c|}{ Multivariable analysis } \\
\hline & & OR $(95 \% \mathrm{CI})$ & $P^{*}$ & aOR $(95 \% \mathrm{CI})$ & $P$ & OR $(95 \% \mathrm{CI})$ & $P^{*}$ & aOR $(95 \% \mathrm{CI})$ & $P$ & OR $(95 \% \mathrm{CI})$ & $P^{*}$ & aOR $(95 \% \mathrm{CI})$ & $P$ \\
\hline & $23 \mathrm{~A}$ & $2 \cdot 00(0 \cdot 63-6 \cdot 38)$ & $0 \cdot 124$ & $1 \cdot 34(0 \cdot 40-4 \cdot 55)$ & 0.635 & $2 \cdot 22(0 \cdot 81-6 \cdot 07)$ & $0 \cdot 119$ & $1.49(0.49-4.52)$ & 0.479 & $1.43(0.42-4.88)$ & $0 \cdot 566$ & $0 \cdot 85(0 \cdot 19-3 \cdot 78)$ & 0.829 \\
\hline & $23 \mathrm{~F}$ & $1 \cdot 50(0 \cdot 55-4 \cdot 09)$ & $0 \cdot 428$ & $1.42(0 \cdot 49-4 \cdot 08)$ & $0 \cdot 517$ & $1 \cdot 04(0 \cdot 46-2 \cdot 39)$ & 0.920 & $0.69(0 \cdot 28-1 \cdot 72)$ & 0.424 & $1.47(0.61-3.57)$ & $0 \cdot 392$ & $0 \cdot 75(0 \cdot 25-2 \cdot 19)$ & 0.592 \\
\hline & 31 & $2.00(0.56-7 \cdot 09)$ & $0 \cdot 283$ & $1.59(0.41-6 \cdot 14)$ & 0.499 & $0 \cdot 73(0 \cdot 21-2 \cdot 48)$ & 0.611 & $0 \cdot 40(0 \cdot 11-1 \cdot 50)$ & $0 \cdot 176$ & $1 \cdot 64(0 \cdot 33-8 \cdot 10$ & $0 \cdot 546$ & $0 \cdot 46(0.08-2 \cdot 67)$ & $0 \cdot 391$ \\
\hline & $33 \mathrm{~F}$ & $2 \cdot 16(0 \cdot 84-5 \cdot 58)$ & $0 \cdot 112$ & $2.33(0.84-6.49)$ & $0 \cdot 104$ & $1 \cdot 20(0.52-2 \cdot 79)$ & 0.672 & $1.08(0.42-2.79)$ & $0 \cdot 876$ & $1.47(0.54-4.05)$ & 0.453 & $1.53(0.43-5.45)$ & 0.515 \\
\hline & $35 \mathrm{~F}$ & $1.38(0 \cdot 35-5 \cdot 49)$ & $0 \cdot 643$ & $0 \cdot 86(0 \cdot 20-3 \cdot 64)$ & $0 \cdot 839$ & $1 \cdot 71(0.53-5.57)$ & $0 \cdot 370$ & $1 \cdot 35(0 \cdot 36-5 \cdot 04)$ & 0.653 & $1.47(0 \cdot 40-5 \cdot 48)$ & 0.564 & $1 \cdot 58(0 \cdot 30-8 \cdot 34)$ & 0.590 \\
\hline \multirow{4}{*}{$\begin{array}{l}\text { Clinical } \\
\text { presentation }\end{array}$} & Other & Ref. & $0 \cdot 005$ & Ref. & - & Ref. & 0.008 & - & - & Ref. & 0.007 & - & - \\
\hline & Meningitis & $0 \cdot 81(0 \cdot 44-1 \cdot 51)$ & $0 \cdot 513$ & $1 \cdot 33(0 \cdot 67-2 \cdot 67)$ & $0 \cdot 416$ & $0 \cdot 44(0 \cdot 25-0 \cdot 79)$ & $0 \cdot 006$ & - & - & $0 \cdot 41(0 \cdot 22-0 \cdot 79)$ & $0 \cdot 007$ & - & - \\
\hline & Pneumonia & $0 \cdot 88(0 \cdot 57-1 \cdot 35)$ & 0.557 & $0.86(0.53-1.40)$ & $0 \cdot 557$ & $0 \cdot 80(0 \cdot 55-1 \cdot 15)$ & $0 \cdot 228$ & - & - & $1.04(0.69-1.55)$ & $0 \cdot 865$ & - & - \\
\hline & Septicaemia & $1.86(1.07-3.23)$ & $0 \cdot 027$ & $1 \cdot 70(0 \cdot 92-3 \cdot 14)$ & 0.091 & $1 \cdot 19(0.72-1.98)$ & $0 \cdot 497$ & - & - & $1.28(0.69-2.37)$ & 0.434 & - & - \\
\hline \multirow[t]{2}{*}{ Alcohol misuse } & No & Ref. & - & - & - & Ref. & - & - & - & Ref. & - & - & - \\
\hline & Yes & $1 \cdot 01(0 \cdot 61-1 \cdot 66)$ & 0.973 & - & - & $0 \cdot 76(0 \cdot 48-1 \cdot 20)$ & $0 \cdot 240$ & - & - & $0.91(0.55-1 \cdot 48)$ & 0.692 & - & - \\
\hline \multirow{2}{*}{$\begin{array}{l}\text { Chronic heart } \\
\text { disease }\end{array}$} & No & Ref. & - & - & - & Ref. & - & - & - & Ref. & - & Ref. & - \\
\hline & Yes & $2.61(1.93-3.53)$ & $<0.001$ & - & - & $3 \cdot 23(2 \cdot 45-4 \cdot 28)$ & $<0.001$ & - & - & $4 \cdot 11(2 \cdot 95-5 \cdot 73)$ & $<0.001$ & $1 \cdot 69(1 \cdot 11-2 \cdot 57)$ & 0.014 \\
\hline \multirow{2}{*}{$\begin{array}{l}\text { Chronic liver } \\
\text { disease }\end{array}$} & No & Ref. & - & - & - & Ref. & - & - & - & Ref. & - & Ref. & - \\
\hline & Yes & $1.89(1.06-3.39)$ & 0.032 & - & - & $1 \cdot 63(0.94-2 \cdot 84)$ & $0 \cdot 084$ & - & - & $1 \cdot 82(0 \cdot 93-3 \cdot 55)$ & 0.079 & $3.34(1.47-7 \cdot 63)$ & 0.004 \\
\hline \multirow{2}{*}{$\begin{array}{l}\text { Chronic lung } \\
\text { disease }\end{array}$} & No & Ref. & - & - & - & Ref. & - & - & - & Ref. & - & Ref. & - \\
\hline & Yes & $1.72(1.27-2.32)$ & $<0.001$ & - & - & $1 \cdot 89(1 \cdot 44-2 \cdot 47)$ & $<0.001$ & - & - & $2 \cdot 79(2 \cdot 05-3 \cdot 81)$ & $<0.001$ & $2.08(1 \cdot 39-3 \cdot 12)$ & $<0.001$ \\
\hline \multirow{2}{*}{$\begin{array}{l}\text { Chronic renal } \\
\text { disease }\end{array}$} & No & Ref. & - & - & - & Ref. & - & Ref. & - & Ref. & - & Ref. & - \\
\hline & Yes & $1.90(1.28-2.82)$ & 0.002 & - & - & $1 \cdot 84(1 \cdot 27-2 \cdot 68)$ & $0 \cdot 001$ & $0 \cdot 61(0 \cdot 38-0 \cdot 99)$ & $0 \cdot 048$ & $1 \cdot 85(1 \cdot 20-2 \cdot 84)$ & $0 \cdot 005$ & $0.55(0 \cdot 31-0.96)$ & 0.036 \\
\hline \multirow[t]{2}{*}{ Diabetes } & No & Ref. & - & - & - & Ref. & - & - & - & Ref. & - & - & - \\
\hline & Yes & $1 \cdot 45(0 \cdot 97-2 \cdot 17)$ & $0 \cdot 072$ & - & - & $1 \cdot 49(1 \cdot 04-2 \cdot 15)$ & 0.031 & - & - & $1.53(1.01-2.34)$ & 0.046 & - & - \\
\hline \multirow{5}{*}{$\begin{array}{l}\text { Immuno- } \\
\text { suppression } \\
\text { No. of risk } \\
\text { factors }\end{array}$} & No & Ref. & - & - & - & Ref. & - & Ref. & - & Ref. & - & Ref. & - \\
\hline & Yes & $1 \cdot 67(1 \cdot 12-2 \cdot 27)$ & $0 \cdot 012$ & - & - & $2 \cdot 94(2 \cdot 06-4 \cdot 23)$ & $<0.001$ & $2.44(1.57-3.81)$ & $<0.001$ & $2 \cdot 77(1 \cdot 77-4 \cdot 34)$ & $<0.001$ & $3.53(2.01-6 \cdot 19)$ & $<0.001$ \\
\hline & 0 & Ref. & $<0.001$ & Ref. & - & Ref. & $<0.001$ & Ref. & - & Ref. & $<0.001$ & - & - \\
\hline & 1 & $2 \cdot 89(2 \cdot 03-4 \cdot 12)$ & $<0.001$ & $2 \cdot 11(1 \cdot 43-3 \cdot 13)$ & $<0.001$ & $2 \cdot 78(2 \cdot 05-3 \cdot 80)$ & $<0.001$ & $1 \cdot 64(1 \cdot 14-2 \cdot 36)$ & 0.008 & $3.41(2 \cdot 46-4.74)$ & $<0.001$ & - & - \\
\hline & $\geqslant 2$ & $3 \cdot 63(2 \cdot 53-5 \cdot 23)$ & $<0.001$ & $2 \cdot 17(1 \cdot 43-3 \cdot 31)$ & $0 \cdot 019$ & $4 \cdot 73(3 \cdot 43-6 \cdot 52)$ & $<0.001$ & $2 \cdot 22(1.43-3.43)$ & $<0.001$ & $5 \cdot 82(4 \cdot 05-8 \cdot 37)$ & $<0.001$ & - & - \\
\hline
\end{tabular}

aOR, Adjusted odds ratio; CI, confidence interval; Ref., reference group.

Values in bold indicate statistical significance $(P<0 \cdot 05)$.

* For reference groups the Wald test $P$ value is reported.

$\dagger$ Included in the multivariable model irrespective of significance. 

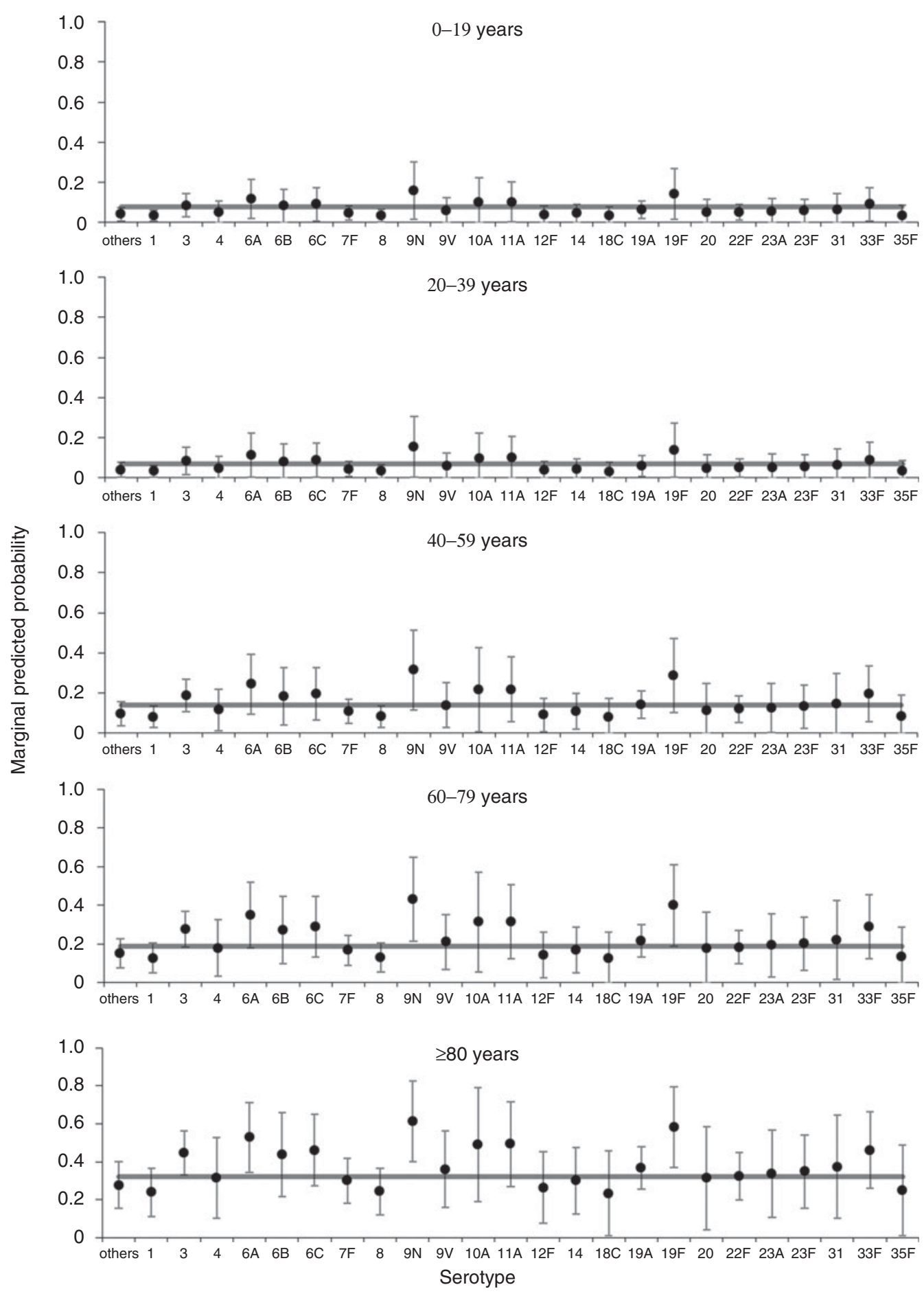

Fig. 2. Predicted marginal probabilities of survival after 30 days following diagnosis with invasive pneumococcal disease by serotype. The grey line indicates the average for each age group. Probabilities are adjusted for sex, deprivation, serotype, clinical presentation and number of risk factors.

by a meta-analysis and systematic review of other studies, including assessment of individual studies with a variety of reference groups, effect sizes and strengths of association [7, 8]. Due to the reduced statistical power for children, the statistical significance of associations between serotype and 30-day mortality is restricted to older adults. When the window following infection was widened to 12 months, only one serotype (19 F) remained associated with increased mortality. At 36 months, no associations between 

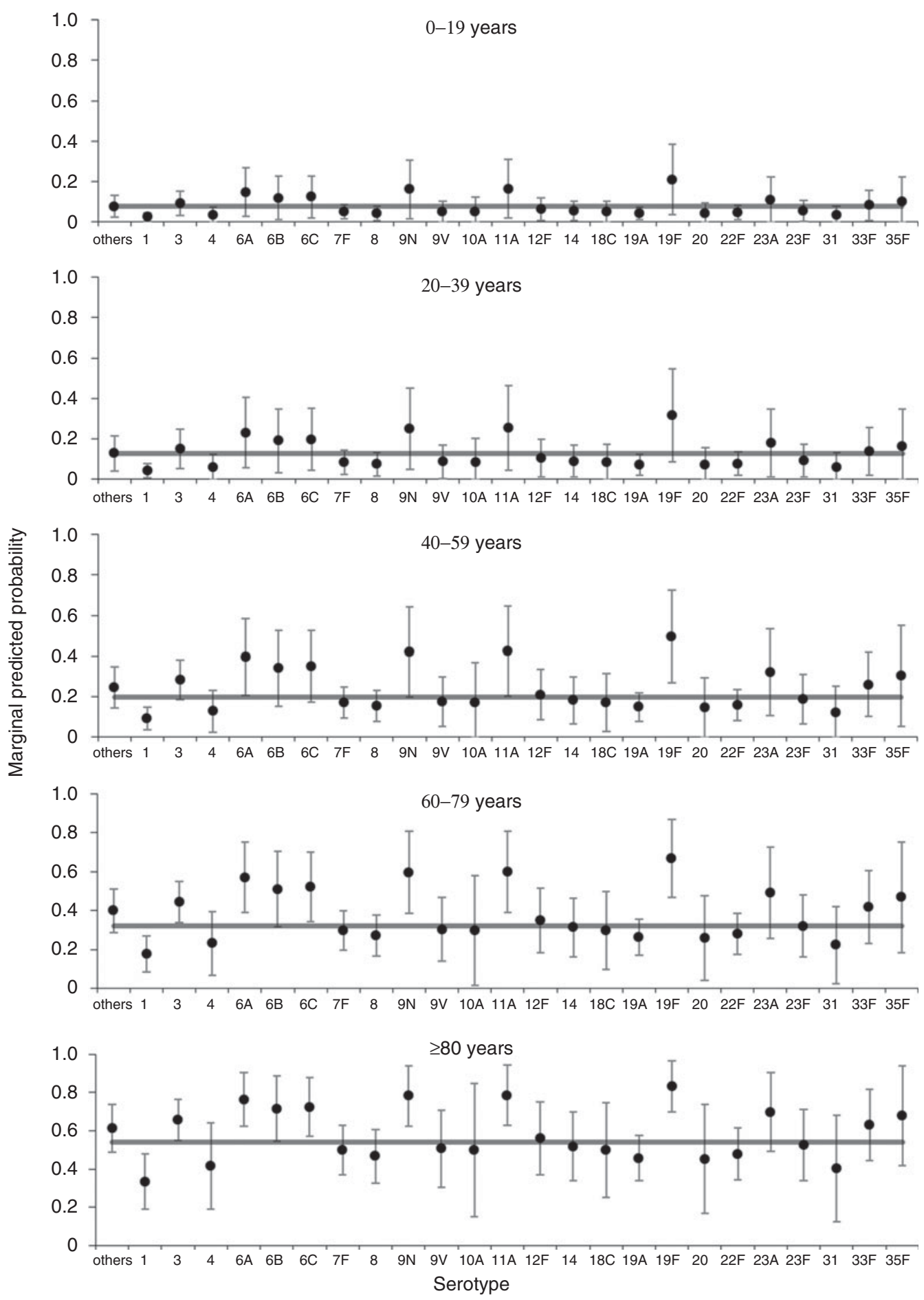

Fig. 3. Predicted marginal probabilities of survival after 12 months following diagnosis with invasive pneumococcal disease by serotype. The grey line indicates the average for each age group. Probabilities are adjusted for sex, serotype and risk factors (number of, chronic renal disease, and immunosuppression).

individual serotypes and increased mortality were found. Statistically significant associations were found for reduced mortality rates associated with serotype 1 (12 and 36 months). This finding is supported by considerable existing evidence for reduced virulence and relative mortality for serotype 1, although the mechanism of this reduced severity has yet to be established $[3,7,8]$. 

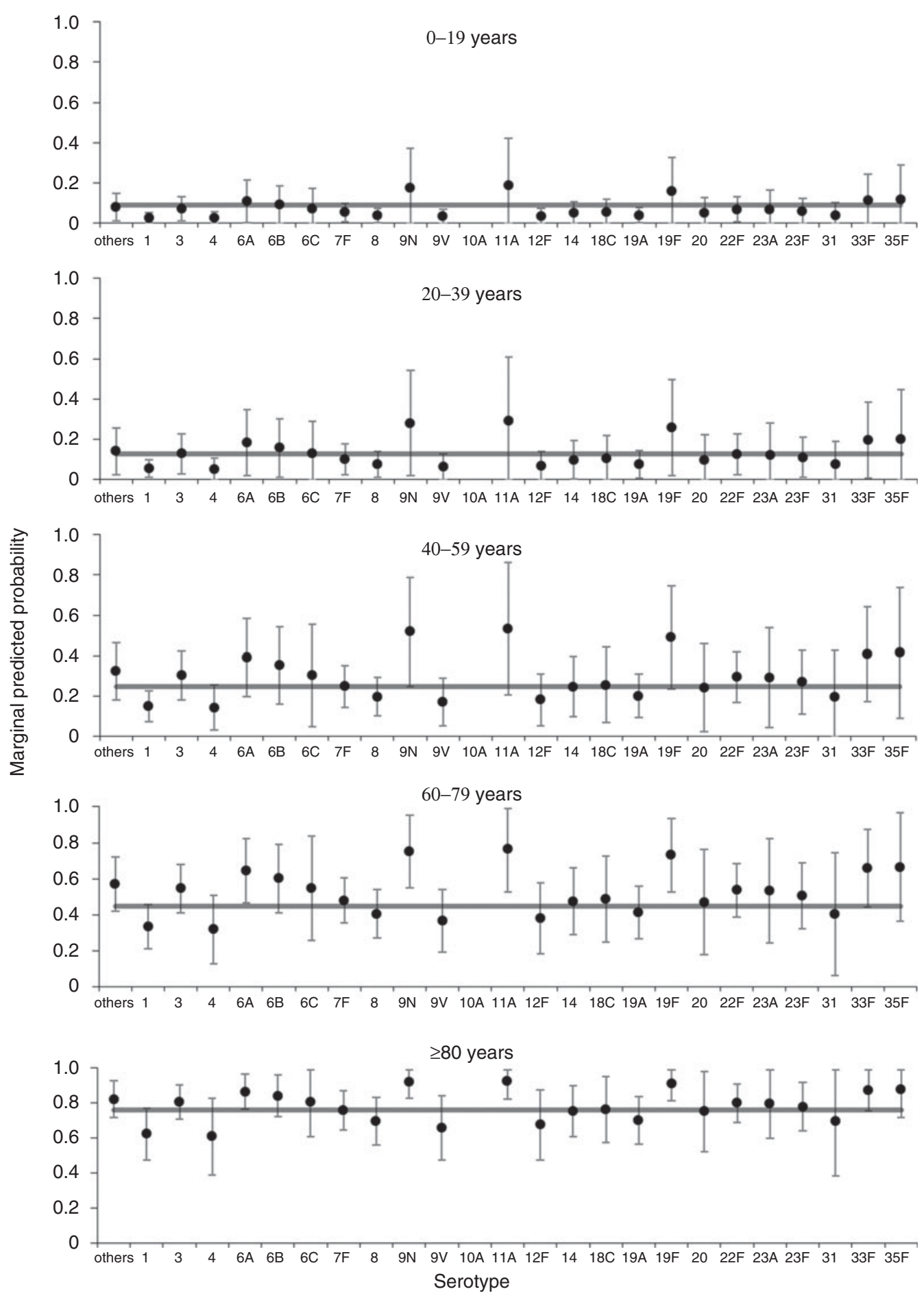

Fig. 4. Predicted marginal probabilities of survival after 36 months following diagnosis with invasive pneumococcal disease by serotype. The grey line indicates the average for each age group. Probabilities are adjusted for sex, pneumococcal vaccination status, deprivation, serotype, and risk factors (chronic liver disease, chronic lung disease, and immunosuppression).

The lack of association between three serotypes ( $3,6 \mathrm{~A}$, $9 \mathrm{~N}$ ) and increased mortality rates using time-frames beyond 30 days suggests that the clinical effects of differential virulence due to these serotypes are restricted to pathogenesis (and/or response to treatment) during the acute stage of the infection. Serotype $19 \mathrm{~F}$ was found to be associated with increased mortality at 12 months as well as 30 days, but not when the window was 
Table 4. Crude and adjusted associations with longer term survival following diagnosis with invasive pneumococcal disease

\begin{tabular}{|c|c|c|c|c|c|c|c|c|c|c|c|}
\hline \multirow[b]{4}{*}{ Variable } & \multirow[b]{4}{*}{ Category } & \multicolumn{6}{|l|}{ All cases } & \multirow{2}{*}{\multicolumn{4}{|c|}{$\begin{array}{l}\text { Cases surviving }>30 \text { days post-diagnosis } \\
\text { Cox proportional hazards model }\end{array}$}} \\
\hline & & & & Cox proportiona & 1 hazards & model & & & & & \\
\hline & & \multicolumn{2}{|c|}{ Log rank test } & \multicolumn{2}{|l|}{ Main effect } & \multicolumn{2}{|c|}{ Time-dependent effect } & \multicolumn{2}{|l|}{ Main effect } & \multicolumn{2}{|c|}{ Time-dependent effect } \\
\hline & & $\chi^{2}$ (D.F.) & $P$ & HR $(95 \% \mathrm{CI})$ & $P^{*}$ & HR $(95 \% \mathrm{CI})$ & $P$ & HR $(95 \%$ CI) & $P^{*}$ & HR $(95 \% \mathrm{CI})$ & $P$ \\
\hline \multirow{5}{*}{ Age group, years $\dagger$} & $0-19$ & $396 \cdot 55$ (4) & $<0.001$ & Ref. & $<0.001$ & - & - & Ref. & $<0.001$ & - & - \\
\hline & $20-39$ & - & - & $1.83(0.87-3.88)$ & $0 \cdot 114$ & - & - & $6 \cdot 96(1 \cdot 46-33 \cdot 12)$ & 0.015 & - & - \\
\hline & $40-59$ & - & - & $1 \cdot 93(0 \cdot 90-4 \cdot 13)$ & $0 \cdot 091$ & $1 \cdot 26(1 \cdot 06-1 \cdot 49)$ & 0.008 & $15 \cdot 06(3 \cdot 61-62 \cdot 83)$ & $<0.001$ & - & - \\
\hline & $60-79$ & - & - & $2.49(1.23-5.06)$ & $0 \cdot 011$ & $1.41(1.20-1.65)$ & $<0.001$ & $38 \cdot 00(9 \cdot 28-155 \cdot 66)$ & $<0.001$ & - & - \\
\hline & $\geqslant 80$ & - & - & $4 \cdot 60(2 \cdot 25-9 \cdot 39)$ & $<0.001$ & $1.48(1.26-1.74)$ & $<0.001$ & $96 \cdot 24(23 \cdot 27-397 \cdot 94)$ & $<0.001$ & - & - \\
\hline \multirow[t]{2}{*}{$\operatorname{Sex} \dagger$} & Female & $1 \cdot 69(1)$ & $0 \cdot 193$ & Ref. & - & - & - & Ref. & - & - & - \\
\hline & Male & - & - & $1 \cdot 18(0 \cdot 88-1 \cdot 60)$ & $0 \cdot 268$ & - & - & $1.82(1 \cdot 42-2 \cdot 33)$ & $<0.001$ & - & - \\
\hline \multirow[t]{7}{*}{ Year of diagnosis } & 2006-2007 & $4 \cdot 49(6)$ & $0 \cdot 611$ & - & - & - & - & - & - & - & - \\
\hline & $2007-2008$ & - & - & - & - & - & - & - & - & - & - \\
\hline & 2008-2009 & - & - & - & - & - & - & - & - & - & - \\
\hline & 2009-2010 & - & - & - & - & - & - & - & - & - & - \\
\hline & 2010-2011 & - & - & - & - & - & - & - & - & - & - \\
\hline & 2011-2012 & - & - & - & - & - & - & - & - & - & - \\
\hline & 2012-2013 & - & - & - & - & - & - & - & - & - & - \\
\hline \multirow[t]{5}{*}{ Deprivation } & Quintile 1 & $12 \cdot 11(4)$ & $0 \cdot 017$ & Ref. & $0 \cdot 006$ & - & - & - & - & - & - \\
\hline & Quintile 2 & - & - & $1.01(0 \cdot 73-1 \cdot 40)$ & $0 \cdot 956$ & - & - & - & - & - & - \\
\hline & Quintile 3 & - & - & $1.27(0.93-1.75)$ & $0 \cdot 138$ & - & - & - & - & - & - \\
\hline & Quintile 4 & - & - & $1 \cdot 60(1 \cdot 18-2 \cdot 17)$ & $0 \cdot 003$ & - & - & - & - & - & - \\
\hline & Quintile 5 & - & - & $1.27(0.93-1.72)$ & $0 \cdot 135$ & - & - & - & - & - & - \\
\hline \multirow{2}{*}{ Immunization } & Vaccinated & $65 \cdot 17(1)$ & $<0.001$ & - & - & - & - & - & - & - & - \\
\hline & Unvaccinated & - & - & - & - & - & - & - & - & - & - \\
\hline \multirow[t]{15}{*}{ Serotype } & Other $\dagger$ & $157 \cdot 50(24)$ & $<0.001$ & Ref. & $0 \cdot 002$ & - & - & Ref. & $<0.001$ & - & - \\
\hline & 1 & - & - & $0.51(0 \cdot 30-0 \cdot 86)$ & $0 \cdot 011$ & - & - & $0 \cdot 32(0 \cdot 16-0 \cdot 63)$ & $0 \cdot 001$ & - & - \\
\hline & 3 & - & - & $1.08(0.73-1.61)$ & $0 \cdot 700$ & - & - & $0.65(0.38-1 \cdot 11)$ & $0 \cdot 117$ & - & - \\
\hline & 4 & - & - & $0 \cdot 77(0 \cdot 40-1 \cdot 48)$ & $0 \cdot 432$ & - & - & $0 \cdot 50(0 \cdot 22-1 \cdot 17)$ & $0 \cdot 109$ & - & - \\
\hline & $6 \mathrm{~A}$ & - & - & $1 \cdot 34(0 \cdot 77-2 \cdot 30)$ & $0 \cdot 297$ & - & - & $0.76(0.34-1.70)$ & $0 \cdot 505$ & - & - \\
\hline & $6 \mathrm{~B}$ & - & - & $1.33(0.74-2.38)$ & $0 \cdot 346$ & - & - & $0.82(0.38-1.76)$ & $0 \cdot 610$ & - & - \\
\hline & $6 \mathrm{C}$ & - & - & $1 \cdot 21(0 \cdot 70-2 \cdot 09)$ & $0 \cdot 487$ & - & - & $0 \cdot 80(0 \cdot 36-1 \cdot 75)$ & $0 \cdot 577$ & - & - \\
\hline & $7 \mathrm{~F}$ & - & - & $0.68(0.43-1.09)$ & $0 \cdot 107$ & - & - & $0.43(0.23-0.80)$ & $0 \cdot 008$ & - & - \\
\hline & 8 & - & - & $0.81(0.52-1 \cdot 27)$ & $0 \cdot 357$ & - & - & $0 \cdot 67(0 \cdot 39-1 \cdot 15)$ & $0 \cdot 150$ & - & - \\
\hline & $9 \mathrm{~N}$ & - & - & $2.37(1.29-4.37)$ & $0 \cdot 007$ & - & - & $1.74(0.67-4.56)$ & $0 \cdot 257$ & - & - \\
\hline & $9 \mathrm{~V}$ & - & - & $0.95(0.55-1.65)$ & $0 \cdot 864$ & - & - & $0 \cdot 60(0 \cdot 30-1 \cdot 19)$ & $0 \cdot 147$ & - & - \\
\hline & $10 \mathrm{~A}$ & - & - & $0.96(0.34-2.71)$ & $0 \cdot 942$ & - & - & $<0 \cdot 1(0 \cdot 00-)$. & $1 \cdot 000$ & - & - \\
\hline & $11 \mathrm{~A}$ & - & - & $1.68(0.96-2.93)$ & $0 \cdot 067$ & - & - & $1.29(0.63-2.68)$ & $0 \cdot 487$ & - & - \\
\hline & $12 \mathrm{~F}$ & - & - & $0.73(0.39-1.35)$ & $0 \cdot 311$ & - & - & $0 \cdot 51(0 \cdot 23-1 \cdot 12)$ & $0 \cdot 093$ & - & - \\
\hline & 14 & - & - & $0.92(0.55-1.55)$ & $0 \cdot 765$ & - & - & $0 \cdot 69(0.37-1 \cdot 29)$ & $0 \cdot 244$ & - & - \\
\hline
\end{tabular}


Table 4 (cont.)

\begin{tabular}{|c|c|c|c|c|c|c|c|c|c|c|c|}
\hline \multirow[b]{4}{*}{ Variable } & \multirow[b]{4}{*}{ Category } & \multicolumn{6}{|l|}{ All cases } & \multirow{2}{*}{\multicolumn{4}{|c|}{$\begin{array}{l}\text { Cases surviving }>30 \text { days post-diagnosis } \\
\text { Cox proportional hazards model }\end{array}$}} \\
\hline & & & & Cox proportiona & hazards & model & & & & & \\
\hline & & \multicolumn{2}{|c|}{ Log rank test } & \multicolumn{2}{|l|}{ Main effect } & \multicolumn{2}{|c|}{ Time-dependent effect } & \multicolumn{2}{|l|}{ Main effect } & \multicolumn{2}{|c|}{ Time-dependent effect } \\
\hline & & $\chi^{2}$ (D.F.) & $P$ & $\operatorname{HR}(95 \% \mathrm{CI})$ & $P^{*}$ & $\operatorname{HR}(95 \% \mathrm{CI})$ & $P$ & $\operatorname{HR}(95 \% \mathrm{CI})$ & $P^{*}$ & $\mathrm{HR}(95 \% \mathrm{CI})$ & $P$ \\
\hline & $18 \mathrm{C}$ & - & - & $1 \cdot 25(0 \cdot 62-2 \cdot 50)$ & $0 \cdot 530$ & - & - & $1 \cdot 53(0 \cdot 66-3 \cdot 54)$ & $0 \cdot 324$ & - & - \\
\hline & $19 \mathrm{~A}$ & - & - & $0 \cdot 80(0.53-1 \cdot 22)$ & $0 \cdot 305$ & - & - & $0 \cdot 45(0 \cdot 26-0 \cdot 80)$ & $\mathbf{0 \cdot 0 0 7}$ & - & - \\
\hline & $19 \mathrm{~F}$ & - & - & $1.64(0.91-2.97)$ & $0 \cdot 100$ & - & - & $0.98(0 \cdot 42-2 \cdot 31)$ & $0 \cdot 971$ & - & - \\
\hline & 20 & - & - & $0 \cdot 70(0 \cdot 31-1 \cdot 57)$ & $0 \cdot 390$ & - & - & $0 \cdot 42(0 \cdot 15-1 \cdot 22)$ & $0 \cdot 112$ & - & - \\
\hline & $22 \mathrm{~F}$ & - & - & $0.83(0.54-1 \cdot 27)$ & $0 \cdot 387$ & - & - & $0.57(0 \cdot 33-0.98)$ & $0 \cdot 044$ & - & - \\
\hline & $23 \mathrm{~A}$ & - & - & $1 \cdot 33(0 \cdot 72-2 \cdot 44)$ & $0 \cdot 358$ & - & - & $1 \cdot 17(0 \cdot 55-2 \cdot 49)$ & $0 \cdot 679$ & - & - \\
\hline & $23 \mathrm{~F}$ & - & - & $0 \cdot 70(0 \cdot 41-1 \cdot 21)$ & $0 \cdot 202$ & - & - & $0 \cdot 47(0 \cdot 24-0 \cdot 91)$ & $0 \cdot 024$ & - & - \\
\hline & 31 & - & - & $0.96(0.48-1.93)$ & $0 \cdot 908$ & - & - & $0.62(0 \cdot 25-1 \cdot 52)$ & $0 \cdot 294$ & - & - \\
\hline & $33 \mathrm{~F}$ & - & - & $1 \cdot 31(0 \cdot 76-2 \cdot 26)$ & $0 \cdot 328$ & - & - & $0 \cdot 86(0 \cdot 43-1 \cdot 74)$ & 0.675 & - & - \\
\hline & $35 \mathrm{~F}$ & - & - & $0.89(0.42-1.90)$ & $0 \cdot 763$ & - & - & $26 \cdot 76(4 \cdot 77-149 \cdot 58)$ & $<0 \cdot 001$ & - & - \\
\hline \multirow[t]{4}{*}{ Clinical presentation } & Other & $27 \cdot 32(3)$ & $<0 \cdot 001$ & Ref. & $0 \cdot 039$ & - & - & Ref. & - & - & - \\
\hline & Meningitis & - & - & $1 \cdot 25(0 \cdot 69-2 \cdot 26)$ & $0 \cdot 469$ & $0 \cdot 78(0 \cdot 67-0 \cdot 91)$ & $0 \cdot 002$ & $0 \cdot 20(0 \cdot 09-0 \cdot 46)$ & $<0 \cdot 001$ & - & - \\
\hline & Pneumonia & - & - & $0.77(0.59-1 \cdot 00)$ & $0 \cdot 052$ & - & - & $0.73(0 \cdot 51-1 \cdot 03)$ & $0 \cdot 072$ & - & - \\
\hline & Septicaemia & - & - & $1 \cdot 02(0 \cdot 72-1 \cdot 45)$ & $0 \cdot 925$ & - & - & $0 \cdot 66(0 \cdot 40-1 \cdot 11)$ & $0 \cdot 121$ & - & - \\
\hline \multirow[t]{2}{*}{ Alcohol misuse } & No & $0 \cdot 01(1)$ & $0 \cdot 916$ & - & - & - & - & - & & - & - \\
\hline & Yes & - & - & - & - & - & - & - & & - & - \\
\hline \multirow[t]{2}{*}{ Chronic heart disease } & No & $132 \cdot 46(1)$ & $<0 \cdot 001$ & Ref. & - & - & - & Ref. & - & - & - \\
\hline & Yes & - & - & $1 \cdot 33(1 \cdot 10-1 \cdot 61)$ & $0 \cdot 004$ & - & - & $1 \cdot 36(1 \cdot 05-1 \cdot 77)$ & $0 \cdot 019$ & - & - \\
\hline \multirow[t]{2}{*}{ Chronic liver disease } & No & $16 \cdot 59(1)$ & $<0 \cdot 001$ & Ref. & - & - & - & Ref. & - & - & - \\
\hline & Yes & - & - & $2 \cdot 30(1 \cdot 62-3 \cdot 27)$ & $<0 \cdot 001$ & - & - & $2 \cdot 88(1 \cdot 78-4 \cdot 65)$ & $<0 \cdot 001$ & - & - \\
\hline \multirow[t]{2}{*}{ Chronic lung disease } & No & $55 \cdot 39(1)$ & $<0 \cdot 001$ & Ref. & - & - & - & Ref. & - & - & - \\
\hline & Yes & - & - & $1 \cdot 29(0 \cdot 85-1 \cdot 96)$ & $0 \cdot 008$ & - & - & $1 \cdot 37(1 \cdot 06-1 \cdot 77)$ & $0 \cdot 015$ & - & - \\
\hline \multirow[t]{2}{*}{ Chronic renal disease } & No & $23 \cdot 77(1)$ & $<0 \cdot 001$ & - & - & - & - & - & - & - & - \\
\hline & Yes & - & - & - & - & - & - & - & - & - & - \\
\hline \multirow[t]{2}{*}{ Diabetes } & No & $4 \cdot 52(1)$ & $0 \cdot 033$ & - & - & - & - & - & - & - & - \\
\hline & Yes & - & - & - & - & - & - & - & - & - & - \\
\hline \multirow[t]{2}{*}{ Immunosuppression } & No & $63 \cdot 00(1)$ & $<0 \cdot 001$ & Ref. & - & - & - & Ref. & - & - & - \\
\hline & Yes & - & - & $1 \cdot 29(0 \cdot 85-1 \cdot 96)$ & $0 \cdot 229$ & $1 \cdot 11(1 \cdot 02-1 \cdot 22)$ & $0 \cdot 020$ & $2 \cdot 53(1.87-3 \cdot 42)$ & $<0 \cdot 001$ & - & - \\
\hline \multirow[t]{3}{*}{ Number of risk factors } & 0 & $193 \cdot 09(2)$ & $<0 \cdot 001$ & - & - & - & - & - & - & - & - \\
\hline & 1 & - & - & - & - & - & - & - & - & - & - \\
\hline & $\geqslant 2$ & - & - & - & - & - & - & - & - & - & - \\
\hline
\end{tabular}

CI, confidence interval; D.F., degrees of freedom; HR, hazard ratio; Ref., reference group.

Values in bold indicate statistical significance $(P<0 \cdot 05)$.

* For reference groups the Wald test $P$ value is reported.

$\dagger$ Included in the multivariable model irrespective of significance. 

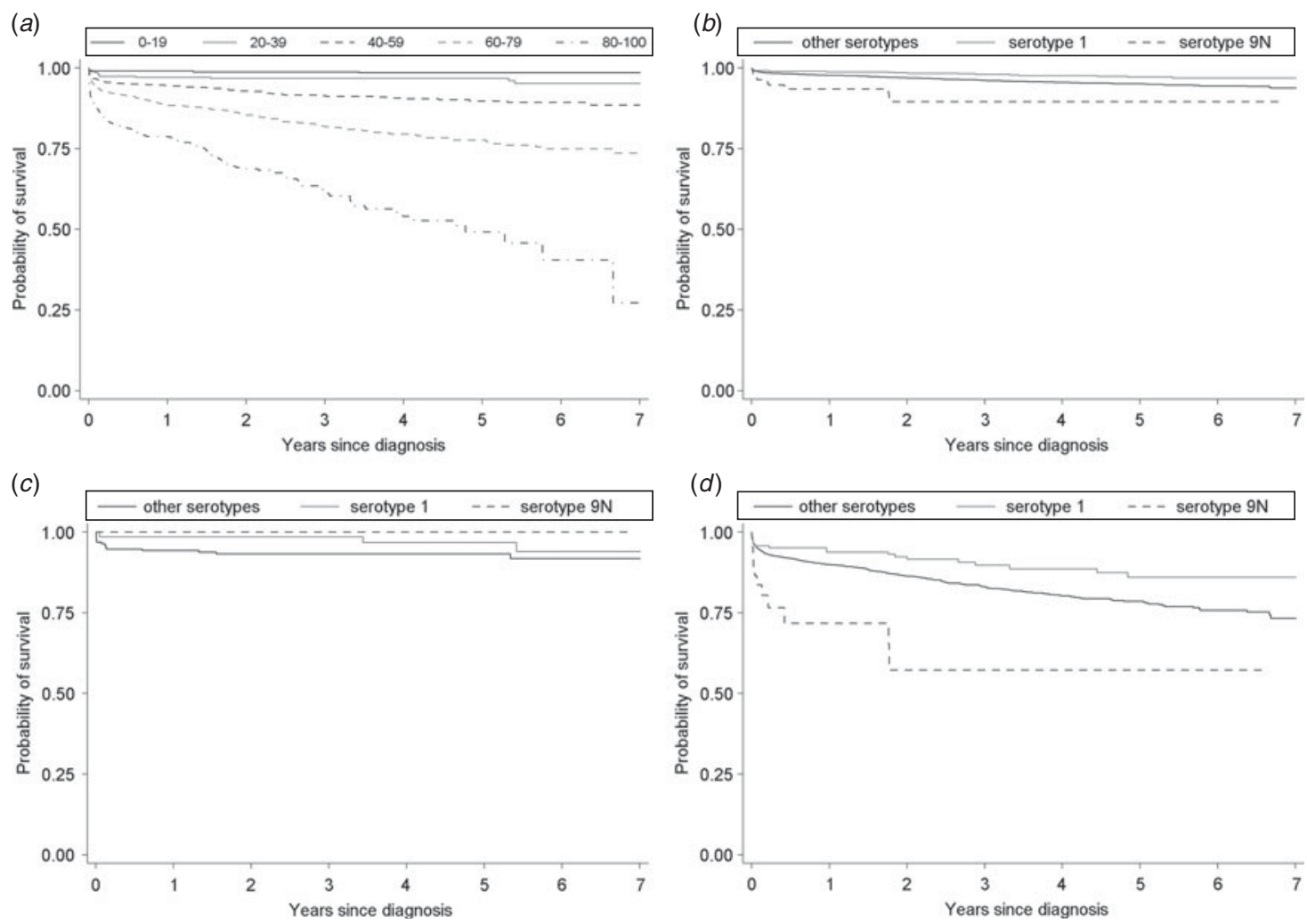

Fig. 5. Adjusted Kaplan-Meier survival curves for time since diagnosis of all cases of invasive pneumococcal disease. (a) All serotypes by age group, $(b)$ significantly associated serotypes for all ages, $(c)$ significantly associated serotypes for ages $0-39$ years, $(d)$ significantly associated serotypes for ages $\geqslant 40$ years. Survival function is adjusted within each age group for sex, deprivation quintile, clinical presentation, chronic heart disease, chronic liver disease, chronic lung disease, and immunosuppression. Only the two serotypes significantly associated with survival from the multivariable model are shown individually.

expanded to 36 months. Furthermore, mortality during the 30 days following infection was significantly associated with the cumulative number of IPD risk factors, rather than individual risk factors being themselves associated with increased mortality, as is seen with the 12 months (immunosuppression) and 36 months (chronic heart disease, chronic liver disease, chronic lung disease, immunosuppression) analysis. It would appear that there is a switch from the combined effect of multiple comorbidities (within 30 days and 12 months of infection) to an effect of specific comorbidities as the time window since infection is expanded, such that within the 36-month time period the number of risk factors is no longer itself significantly associated with mortality. The association between reduced mortality at 12 and 36 months and chronic renal disease may reflect increased contact rates with health services that minimizes the probability of late diagnosis associated with poorer outcomes.

Through the use of a multivariable Cox PH model we have produced fully adjusted, independent associations between serotype and longer-term mortality following IPD. As was seen with the longer window time-point analyses, specific risk factors are predictive of increased mortality rates, rather than the cumulative number. Over a time period of $\leqslant 7$ years, we have shown that serotype is associated with significant variation in mortality with one serotype associated with significantly reduced mortality (1) and one serotype associated with significantly increased mortality $(9 \mathrm{~N})$. Due to a limited statistical power, these effects are only observable in older adults and higher-powered further studies are required to investigate these associations for children and younger adults. Both serotypes 1 and $9 \mathrm{~N}$ have been previously shown to be associated with decreased and increased mortality, respectively [7, 8].

When survival analysis was limited to an observation period that excluded the first 30 days following diagnosis, an altered pattern of serotype-specific mortality rates was observed, with a greater number of serotypes associated with significantly lower mortality rates (serotypes 1, 7F, 19A, 22 F, 23 F) and a 
different serotype associated with significantly higher mortality rates (serotype $35 \mathrm{~F}$ ). However, the estimate for serotype $35 \mathrm{~F}$ has quite low precision and this statistical uncertainty requires further investigation with a larger dataset. Although the association between serotype $9 \mathrm{~N}$ and increased mortality is no longer statistically significant, the point estimate for the HR remains $>1$ and the lack of significance may be due to the reduced power of the analysis compared to the full survival analysis.

We have not been able to consider the influence of events which have occurred post-diagnosis with IPD on the mortality of cases. Of course, it is expected that factors which might contribute towards an increased risk of mortality will have occurred in the follow-up period. However, for these factors to confound relative associations between mortality and individual serotypes they would need to be both causes of increased mortality and be associated with individual serotypes, and it is difficult to foresee situations when this would occur. Furthermore, it should be considered that this study has only assessed relative associations between serotypes and mortality following IPD; no inferences can be made regarding mortality following infection with individual serotypes compared to mortality for similar individuals who have not had IPD.

This study provides the first evidence that the pneumococcal serotype causing IPD is associated with differential longer-term mortality. Due to limited statistical power for children and younger adults it is currently not clear whether these associations apply to these age groups as well as to older adults. Further studies are required to determine the influence of serotype-specific longer-term mortality on the burden of IPD and the consequent implications for the relative benefit of different vaccine compositions.

\section{SUPPLEMENTARY MATERIAL}

For supplementary material accompanying this paper visit http://dx.doi.org/10.1017/S0950268816000856.

\section{ACKNOWLEDGEMENTS}

This work was supported by a grant from the Health Protection Agency Strategic Research and Development Fund and an unrestricted educational grant from Sanofi Pasteur MSD (UK12C1036). We thank the Public Health England North East Health Protection Team for their participation in IPD enhanced surveillance, the Public Health England
Respiratory and Systemic Infections Laboratory for providing serotype results, and all North East NHS microbiology laboratories for reporting cases of IPD.

\section{DECLARATION OF INTEREST}

None.

\section{REFERENCES}

1. O'Brien KL, et al. Burden of disease caused by Streptococcus pneumoniae in children younger than 5 years: global estimates. Lancet 2009; 374: 893-902.

2. Drijkoningen JJC, Rohde GGU. Pneumococcal infection in adults: burden of disease. Clinical Microbiology and Infection 2014; 20 (Suppl. 5): 45-51.

3. Hausdorff WP, Feikin DR, Klugman KP. Epidemiological differences among pneumococcal serotypes. Lancet Infectious Diseases 2005; 5: 83-93.

4. van Hoek AJ, et al. Effect of serotype on focus and mortality of invasive pneumococcal disease: coverage of different vaccines and insight into non-vaccine serotypes. PLoS One 2012; 7: e39150.

5. Harboe ZB, et al. Pneumococcal serotypes and mortality following invasive pneumococcal disease: a populationbased cohort study. PLoS Medicine 2009; 6: e1000081.

6. Martens P, et al. Serotype-specific mortality from invasive Streptococcus pneumoniae disease revisited. BMC Infectious Diseases 2004; 4: 21.

7. Weinberger DM, et al. Association of serotype with risk of death due to pneumococcal pneumonia: a meta-analysis. Clinical Infectious Diseases 2010; 51: 692-699.

8. Grabenstein JD, Musey LK. Differences in serious clinical outcomes of infection caused by specific pneumococcal serotypes among adults. Vaccine 2014; 32: 2399-2405.

9. Navarro-Torné A, et al. Risk factors for death from invasive pneumococcal disease. Emerging Infectious Diseases 2015; 21: 417-425.

10. Sandvall B, Rueda AM, Musher DM. Long-term survival following pneumococcal pneumonia. Clinical Infectious Diseases 2013; 56: 1145-1146.

11. Roed C, et al. Long-term mortality in patients diagnosed with meningococcal disease: a Danish nationwide cohort study. PLoS One 2010; 5: e9662.

12. Wang HE, et al. Long-term mortality after communityacquired sepsis: a longitudinal population-based cohort study. BMJ Open 2014; 4: e004283.

13. Office for National Statistics. Regional profiles: key statistics - North East, August 2012 (http://www.ons.gov.uk/ ons/dcp171780_228225.pdf). Accessed 6 January 2015.

14. Office for National Statistics. General health in England and Wales, 2011 and comparison with 2001 (http:// www.ons.gov.uk/ons/dcp171776_296871.pdf). Accessed 6 January 2015.

15. McLennan D, et al. The English indices of deprivation 2010 (https://www.gov.uk/government/uploads/system/ 
uploads/attachment_data/file/6320/1870718.pdf). Accessed 6 January 2015

16. Chapman KE, Wilson D, Gorton R. Serotype dynamics of invasive pneumococcal disease post-PCV7 and prePCV13 introduction in North East England. Epidemiology and Infection 2013; 141:344-352.
17. Chapman KE, Wilson D, Gorton R. Invasive pneumococcal disease and socioeconomic deprivation: a population study from the North East of England. Journal of Public Health (Oxford) 2013; 35: 558-569.

18. Hosmer DW, Lemeshow S. Applied Logistic Regression. New York: John Wiley \& Sons, 2001. 